\title{
Comparable patencies of the radial artery and right internal thoracic artery or saphenous vein beyond 5 years: Results from the Radial Artery Patency and Clinical Outcomes trial
}

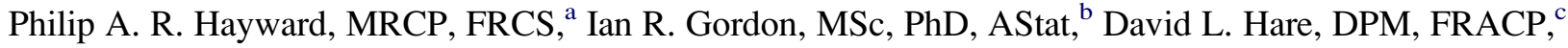 \\ George Matalanis, FRACS, ${ }^{\mathrm{a}}$ Mark L. Horrigan, FRACP, ${ }^{\mathrm{c}}$ Alexander Rosalion, FRACS, ${ }^{\mathrm{a}}$ and \\ Brian F. Buxton, FRACS, FRCS, FRCS $(C)^{\mathrm{a}}$
}

Objective: To investigate the optimum conduit for coronary targets other than the left anterior descending artery, we evaluated long-term patencies and clinical outcomes of the radial artery, right internal thoracic artery, and saphenous vein through the Radial Artery Patency and Clinical Outcomes trial.

\begin{abstract}
Methods: As part of a 10-year prospective, randomized, single-center trial, patients undergoing primary coronary surgery were allocated to the radial artery $(n=198)$ or free right internal thoracic artery $(n=196)$ if aged less than 70 years (group 1$)$, or radial artery $(n=113)$ or saphenous vein $(n=112)$ if aged at least 70 years (group 2$)$. All patients received a left internal thoracic artery to the left anterior descending, and the randomized conduit was used to graft the second largest target. Protocol-directed angiography has been performed at randomly assigned intervals, weighted toward the end of the study period. Grafts are defined as failed if there was occlusion, string sign, or greater than $80 \%$ stenosis, independently reported by 3 assessors. Analysis is by intention to treat.
\end{abstract}

Results: At mean follow up of 5.5 years, protocol angiography has been performed in groups 1 and 2 in 237 and 113 patients, respectively. There are no significant differences within each group in preoperative comorbidity, age, or urgency. Patencies were similar for either of the 2 conduits in each group (log rank analysis, $P=.06$ and $P=.54$, respectively). The differences in estimated 5-year patencies were $6.6 \%$ (radial minus right internal thoracic artery) in group 1 and $2.9 \%$ (radial minus saphenous vein graft) in group 2.

Conclusion: At mean 5-year angiography in largely asymptomatic patients, the selection of arterial or venous conduit for the second graft has not significantly affected patency. This finding offers surgeons, for now, enhanced flexibility in planning revascularization. (J Thorac Cardiovasc Surg 2010;139:60-7)

Earn CME credits at

http://cme.ctsnetjournals.org

The long-term durability of revascularization by coronary artery bypass grafting has been well demonstrated. It stands in contrast to the demonstrably higher requirements for reintervention after percutaneous coronary intervention ${ }^{1,2}$ and provides the basis on which a patient with coronary disease may be referred for surgery despite its greater invasiveness.

From the Department of Cardiac Surgery, ${ }^{\text {a }}$ Austin Hospital and University of Melbourne, the Statistical Consulting Centre, ${ }^{\mathrm{b}}$ University of Melbourne, Parkville, and the Department of Cardiology, ${ }^{\mathrm{c}}$ Austin Hospital and University of Melbourne, Melbourne, Victoria, Australia.

Read at the Eighty-eighth Annual Meeting of The American Association for Thoracic Surgery, San Diego, Calif, May 10-14, 2008.

Received for publication May 6, 2008; revisions received Sept 2, 2009; accepted for publication Sept 28, 2009.

Address for reprints: Brian F. Buxton, FRACS, FRCS, FRCS(C), Victorian Heart Centre, Epworth Hospital, Bridge Road, Richmond, Victoria, Australia (E-mail: brianbuxton@ozemail.com.au).

0022-5223/\$36.00

Copyright (c) 2010 by The American Association for Thoracic Surgery doi:10.1016/j.jtcvs.2009.09.043
The Radial Artery Patency and Clinical Outcomes (RAPCO) trial has been designed to identify the place of the radial artery (RA) in the hierarchy of conduit options available to the modern surgeon to supplement the gold standard left internal thoracic artery (LITA) to left anterior descending (LAD) graft. Patients recruited to this study, monitored by annual clinical review and angiographic follow-up at defined time points, represent the ideal model in which to correlate graft patency with their excellent longterm survival and event-free survival reported previously., 3,4 The midterm to long-term graft patencies in this trial may also further refine our understanding of graft outcomes with optimized modern secondary medical prevention.

\section{PATIENTS AND METHODS}

The prospective approval for this trial from the Hospital Committee of Human Ethics in Research, enrolment criteria, randomization, conduct of surgery, and clinical or angiographic follow-up patients for the RAPCO study has been described at length elsewhere. ${ }^{5}$ In summary, this is a pair of parallel randomized trials with patients less than 70 years of age randomized to receive either an RA or a free right internal thoracic artery (RITA) for the largest coronary target other than the LAD, and older patients randomized to RA or saphenous vein (SV) to a similar target. The target vessel for the randomized conduit was required to have a diameter of at least $1.5 \mathrm{~mm}$, with an inflow stenosis exceeding $70 \%$ and absence of diffuse distal disease. It was prospectively selected by review of the preoperative angiogram and was the largest artery, other than the LAD, meeting the aforementioned 


$$
\begin{aligned}
& \text { Abbreviations and Acronyms } \\
& \begin{aligned}
\text { CI } & =\text { confidence interval } \\
\text { LAD } & =\text { left anterior descending coronary artery } \\
\text { LITA } & =\text { left internal thoracic artery } \\
\text { RA } & =\text { radial artery } \\
\text { RAPCO } & \text { Radial Artery Patency and Clinical } \\
& \text { Outcomes } \\
\text { RITA } & =\text { right internal thoracic artery } \\
\text { SV } & =\text { saphenous vein }
\end{aligned}
\end{aligned}
$$

criteria. If several targets were eligible, an estimation of the magnitude of territory of runoff was used for preoperative or intraoperative final confirmation of the appropriate study target. All patients received a LITA graft to the $\mathrm{LAD}$, and third- and fourth-order grafts were in accordance with the surgeon's preference. All proximal anastomoses were to be placed to the aorta, but 4 were anastomosed to other grafts close to the aorta to form $\mathrm{Y}$ grafts with a short common stem when the conduit length of the study graft was inadequate to reach the aorta comfortably.

A program of postoperative angiography assigned patients to predetermined, randomly allocated intervals varying between 1 month and 10 years from surgery, weighted toward the second half of the study period. In addition to their programmed angiogram, all patients were offered a supplementary midterm angiogram at 5 years. Annual clinical review by a surgeon, cardiologist, and nurse consultant assigned to the trial, together with consultation of the National Death Index, were used to gather information regarding survival and event-free survival. All clinical and angiographic data were prospectively compiled in a Visual FoxPro 5.0 database (Microsoft Corporation, Seattle, Wash) by a dedicated data manager. Preoperative demographic data were compared by Fisher's exact test and estimations of graft patency at time intervals up to 10 years were expressed by generalized Kaplan-Meier plots and interval censoring. All analyses were verified by a university statistician using SPSS software (SPSS, Inc, Chicago, Ill). The analysis of patency was carried out using the intention-to-treat principle: outcomes were analyzed according to the graft to which the patient was randomized. The data are interval censored ${ }^{6}$ : all grafts found to be occluded were discovered in that state at the first protocol-directed angiogram (left censoring). Patients with patent grafts were taken to be right censored at the most recent protocol-directed angiogram. Some patients had more than one protocol-directed angiogram, but no patient in either of the 2 groups had a graft found to be patent at one angiogram and occluded at the next. Nonparametric methods for interval censored data were used. The generalized Kaplan-Meier curve was produced using the method of Turnbull $^{7}$ and the generalized log-rank test ${ }^{8}$; the plot was produced in Minitab (Minitab Statistical Software; Minitab Inc, State College, Pa) and the test was carried out in R (R Foundation for Statistical Computing, Vienna, Austria, using the interval procedure of Fay and Shaw, 2009 [personal communication]). Importantly, the graph and method used here do not assume that graft occlusion occurred at any particular time point (eg, the midpoint) between the operation and the postoperative angiogram. Any such assumption has potential for misleading results. ${ }^{9}$

\section{RESULTS}

The assessment, recruitment, randomization, and followup of patients in the RAPCO trial, in line with the CONSORT criteria, are depicted in Figure 1, $A$ and $B$. Of 1882 patients assessed, 619 were randomized in the RAPCO trial. Forty patients (29 in group 1 and 11 in group 2) did not receive the randomized conduit as an independent graft, primarily owing to lack of conduit of acceptable quality for the study graft or to a general paucity of conduit availability requiring use of sequential grafting (which invalidated the study graft data), owing to surgeon preference, for reasons that are not clear from the data. Among 29 cases in which randomization was not followed in group 1, there were 15 crossovers and 14 usages of a non-trial conduit or sequential technique. In group 2 there were 11 protocol violations, including 7 crossovers. All 40 cases are included in the analysis by intention to treat. Angiographic follow-up has been performed to date as per randomization of timing of restudy in all but 2 patients; one was lost to all follow-up and the other declined angiography. The mean duration of followup was 5.5 years, with a range of 0.1 years to 11.2 years.

The preoperative demographic details of the patients in the 2 groups in the trial are recorded in Tables 1 and 2 . These detail separately patients whose graft patency has been assessed to date and the entire randomized cohort from which they are drawn, for comparison, to confirm that the group whose protocol angiogram has been performed to date is representative of the whole. The distributions of age, gender, cardiovascular risk factors, urgency of surgery, and number of grafts performed are comparable in the 2 arms within each group, except differences in the proportion of elective surgery in group $2(66 \%$ vs $83 \%)$ and in the mean number of grafts per patient (3.1 vs 3.3), which trend toward significance. Other potential explanatory variables such as peripheral vascular disease may be important but were not consistently recorded and are therefore excluded from the analysis. It is noteworthy that the majority of patients had 3-vessel disease, with the mean number of grafts 3.0 or greater in all of the 4 arms in this trial.

The estimated patency of the RA and free RITA in group 1 is depicted in Figure 2. Generalized Kaplan-Meier estimates of graft patency, by intention to treat, in group 1 at 5 years were estimated to be $89.8 \%$ in the radial group (95\% confidence interval [CI], $71.0 \%-100 \%)$ and $83.2 \%$ $(95 \%$ CI, $54.1 \%-100 \%)$ in the RITA group. The absolute difference between the 5-year patencies (RA minus RITA) was estimated to be $6.6 \%$ (95\% CI, $-28.2 \%$ to $41.5 \%$ ). There is no demonstrable difference in the patency of each conduit regardless of analysis by intention to treat $(P=$ .06 ) or by conduit used, although the numbers at risk beyond 7 years are small. There were 5 and 10 occluded grafts and 5 and 6 string signs in the randomized RA and RITA arms, respectively. Four of the failed grafts in the group randomized to RITA were in fact crossovers. Estimates of patency in patients who received the correct randomized conduit show no such trend to difference between RA and RITA $(P=.28)$.

Similar generalized Kaplan-Meier curves for group 2, comparing the RA with SV, are shown in Figure 3. Estimates 


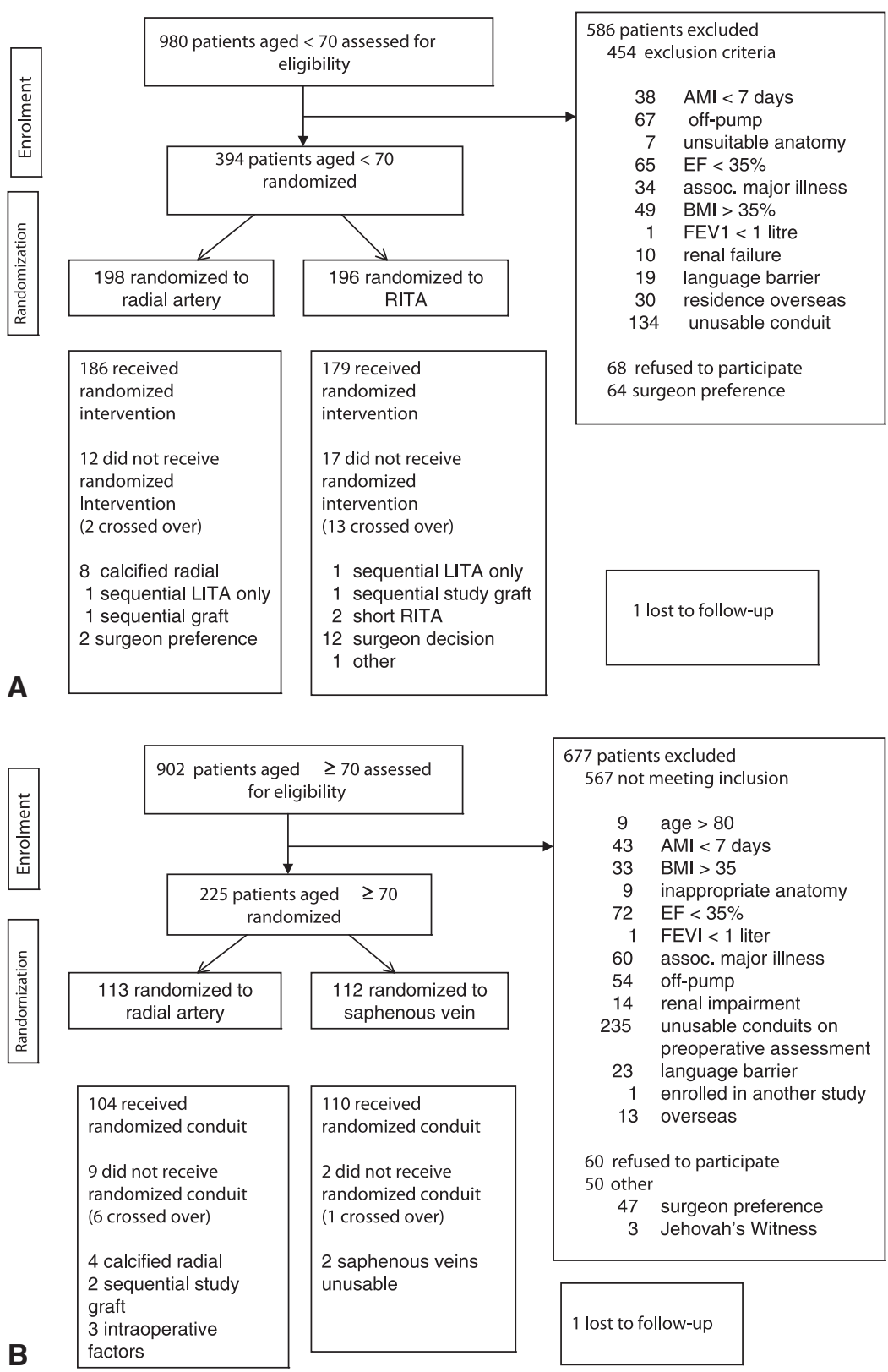

FIGURE 1. A and B, Recruitment, exclusion, randomization, and follow-up in groups 1 and 2 , respectively. RITA, Right internal thoracic artery; LITA, left internal thoracic artery; $A M I$, acute myocardial infarction; $E F$, ejection fraction; $B M I$, body mass index; $F E V_{1}$, forced expiratory volume in 1 second.

of graft patency at 5 years, by intention to treat, in group 2 were estimated to be $90.0 \%$ in the radial group $(95 \% \mathrm{CI}$, $69.0 \%-100 \%)$ and $87.0 \%(95 \%$ CI, $61.4 \%-100 \%)$ in the SV group. The difference between the 5-year patencies (RA minus SV) was estimated to be $2.9 \%$ (95\% CI, $-28.8 \%$ to $34.5 \%$ ). There is, therefore, no demonstrable difference in the patency estimates of these conduits in patients aged over 70 years (log-rank, $P=.29$ ), regardless of analysis by intention to treat or by conduit used. Analysis by conduit used also reveals no difference in patency $(P=$ .40). Inasmuch as there was substantial weighting of the timing of angiography toward the second half of the study period in this group, the proportion of patients who have undergone restudy at more than 5 years remains small, with low numbers at risk as a result. To date, $1 \mathrm{SV}$ has a highgrade in-conduit stenosis and 7 others are occluded. Of the RAs, 3 are occluded ( 1 of which is a crossover) and 2 have string sign but none has a stenosis within the conduit.

\section{DISCUSSION}

The 5-year (and beyond) patencies in conduits used to supplement the LITA-LAD graft suggest that the second 
TABLE 1. Preoperative demographic and operative data for group 1 (aged $<70$ years)

\begin{tabular}{lccccc}
\hline & $\begin{array}{c}\text { RA restudied by } \\
\text { angiogram }(\mathbf{n}=\mathbf{1 2 3})\end{array}$ & $\begin{array}{c}\text { RA enrolled } \\
(\mathbf{n}=\mathbf{1 9 8})\end{array}$ & $\begin{array}{c}\text { RITA restudied by } \\
\text { angiogram }(\mathbf{n}=\mathbf{1 1 4})\end{array}$ & $\begin{array}{c}\text { RITA enrolled } \\
(\mathbf{n}=\mathbf{1 9 6})\end{array}$ & $\begin{array}{c}\text { P value (RA restudied vs } \\
\text { RITA restudied) }\end{array}$ \\
\hline Age, y (mean and range) & $59.6(42.3-71.0)$ & $59.2(37.9-71.0)$ & $59.1(36.2-70.8)$ & $59.5(36.2-70.9)$ & $.96^{*}$ \\
Gender (male) & $111(90 \%)$ & $175(88 \%)$ & $109(95 \%)$ & $178(91 \%)$ & $.13 \dagger$ \\
Diabetes (any) & $10(9 \%)$ & $22(11 \%)$ & $9(7 \%)$ & $20(10 \%)$ & $.96 \dagger$ \\
Hypertension & $64(51 \%)$ & $112(57 \%)$ & $57(51 \%)$ & $99(51 \%)$ & $.80 \dagger$ \\
Elective presentation & $96(77 \%)$ & $160(81 \%)$ & $86(76 \%)$ & $161(82 \%)$ & $.65 \dagger$ \\
Smoking (ever smoked) & $92(73 \%)$ & $151(75 \%)$ & $87(77 \%)$ & $137(70 \%)$ & $.88 \dagger$ \\
No. of grafts (mean \pm SD) & $3.0 \pm .7$ & $3.1 \pm .8$ & $3.2 \pm .8$ & $3.2 \pm .9$ & $.19 *$ \\
Distribution of target & $64: 33: 3$ & $62: 35: 3$ & $70: 25: 5$ & $69: 26: 5$ & $.29 \ddagger$ \\
$\quad$ vessels for study graft & & & & & \\
$\quad$ Cx: RCA: diagonal $(\%))$ & & & & & \\
\hline
\end{tabular}

$R A$, Radial artery; $R I T A$, right internal thoracic artery; $S D$, standard deviation; $C x$, circumflex artery; $R C A$, right coronary artery. RA restudied versus RA enrolled: $P=$ not significant, all variables; RITA restudied versus RITA enrolled: $P=$ not significant, all variables. * $t$ test; $\nmid$ Fisher’s exact test; $\ddagger$ Pearson’s test.

best target may be grafted equally satisfactorily with an RA or free ITA in younger patients and an RA or SV in older patients, with similar angiographic outcomes. $\mathrm{We}^{3,4}$ have previously reported clinical outcomes for both groups with mean 5- or 6-year follow-up, demonstrating equivalent survival and event-free survival in each group irrespective of the conduit used. It is gratifying to see that these excellent clinical outcomes have been matched by high patency rates beyond 5 years, although it was never presumed that this would necessarily be so inasmuch as many patients will survive, even symptom-free, despite graft failures.

The clinical relevance of 5-year patency may lie in patients whose life expectancy is short. One could argue that the majority of graft failures might be expected to occur beyond 5 years and that therefore only the final 10-year patency results in this study will represent a clinically important outcome. However, the increasing proportion of referrals of very elderly patients or of patients with severe comorbidities such as malignancy, severe pulmonary disease, or major arteriopathy has led to a growing population of surgical patients in whom 5-year outcome is all that realistically should be considered. Furthermore, it is the comorbid conditions in these patients that may in fact define the usability of conduits. The finding of comparable patencies in both groups, together with previously reported equal clinical outcomes, allows the surgeon freedom to match the choice of conduit to the patient's other conditions, with the evidence suggesting that this selection will not compromise the midterm success of the coronary operation. Clearly, for younger or fitter patients whose life expectancy is anticipated to be a decade or more, full 10-year patency and clinical outcome data from this trial will be essential before the optimum revascularization strategy can be defined.

$\mathrm{We}^{3}$ have discussed elsewhere that the free RITA was included in this trial for 2 reasons. First, we did not wish to compromise the use of the gold standard LITA-LAD graft and an in situ RITA could not reach all targets. Second, RAPCO was designed as a biological comparison of different conduits used in a similar manner; thus the RITA was used as a free graft as the RA or SV would be. Subsequently, however, we have been persuaded of the benefits of bilateral in situ ITA grafting ${ }^{10,11}$; nonetheless the finding of excellent angiographic and clinical outcomes with the free graft adds an extra layer of flexibility in view of its capacity to reach a wider spectrum of targets than an in situ graft.

TABLE 2. Preoperative demographic and operative data for group 2 (aged $>70$ years)

\begin{tabular}{|c|c|c|c|c|c|}
\hline & $\begin{array}{l}\text { RA restudied } \\
\quad(\mathbf{n}=\mathbf{5 3}) \\
\end{array}$ & $\begin{array}{c}\text { RA enrolled } \\
(n=113) \\
\end{array}$ & $\begin{array}{l}\text { SV restudied } \\
\quad(n=60)\end{array}$ & $\begin{array}{c}\text { SV enrolled } \\
(\mathbf{n}=\mathbf{1 1 2})\end{array}$ & $\begin{array}{c}P \text { value (RA restudied vs } \\
\text { SV restudied) }\end{array}$ \\
\hline Age, y (mean and range) & $73.4(61.4-81.5)$ & $72.5(61.0-83.5)$ & $72.9(60.5-80.6)$ & $73.1(60.5-80.7)$ & $.59 *$ \\
\hline Gender (male) & $44(80 \%)$ & $91(81 \%)$ & $51(86 \%)$ & $91(81 \%)$ & $.80 \dagger$ \\
\hline Diabetes (any) & $16(29 \%)$ & $50(44 \%)$ & $23(39 \%)$ & $52(46 \%)$ & $.43 \dagger$ \\
\hline Hypertension & $23(47 \%)$ & $67(59 \%)$ & $37(61 \%)$ & $77(69 \%)$ & $.06 \dagger$ \\
\hline Elective presentation & $35(71 \%)$ & $87(77 \%)$ & $50(81 \%)$ & $91(81 \%)$ & $.05 \dagger$ \\
\hline Smoking (ever smoked) & $37(73 \%)$ & $75(66 \%)$ & $42(71 \%)$ & $76(68 \%)$ & $.98 \dagger$ \\
\hline No. of grafts $($ mean $\pm \mathrm{SD})$ & $3.1 \pm .8$ & $3.2 \pm .9$ & $3.3 \pm .7$ & $3.3 \pm .7$ & $.05^{*}$ \\
\hline $\begin{array}{l}\text { Distribution of target } \\
\text { vessels for study graft } \\
\text { (Cx: RCA: diagonal }(\%)\end{array}$ & $73: 25: 2$ & 70: 25: & 58: $37:$ & $60: 37: 2$ & $.31 \ddagger$ \\
\hline
\end{tabular}




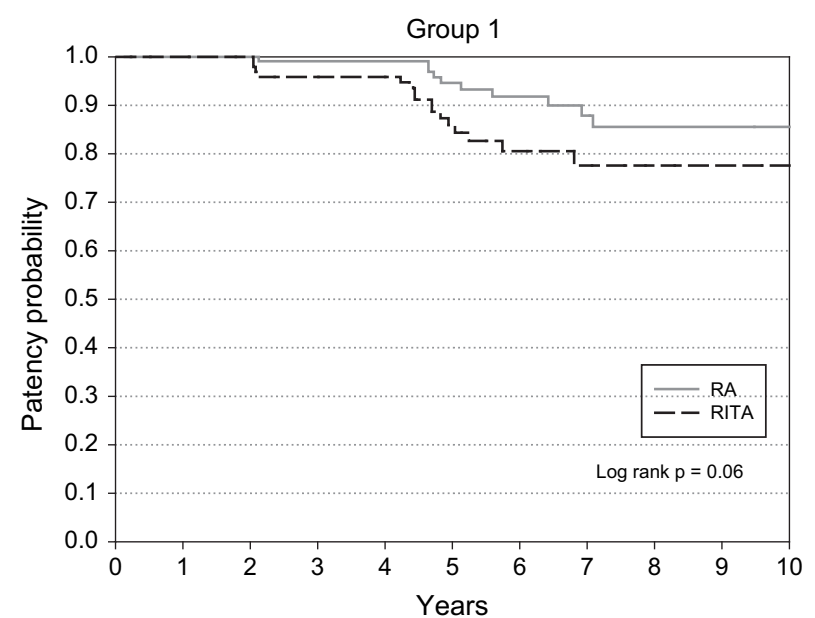

\begin{tabular}{|l|r|r|r|r|r|r|r|r|r|r|r|}
\hline RA & 123 & 118 & 110 & 105 & 101 & 76 & 52 & 39 & 23 & 20 & 4 \\
\hline RITA & 114 & 104 & 97 & 88 & 87 & 56 & 32 & 23 & 14 & 12 & 8 \\
\hline
\end{tabular}

FIGURE 2. Kaplan-Meier estimates of graft patency in group 1 by intention to treat. $R A$, Radial artery; RITA, right internal thoracic artery.

A striking finding is the patency of SV grafts beyond 5 years, which exceeds that described in historical series. ${ }^{12-14}$ It seems likely that rigorous application of secondary prevention methods and modification of lifestyle factors has had a powerful effect on patency of vein grafts over the past 20 years. Surgically, an enhanced understanding of the importance of careful conduit handling, avoidance of overdistention, and discarding of any segments of RA or vein of modest quality may have led to an improvement in the technical conduct of procedures using arterial and venous grafts. If a controlled elective setting may favor superior surgical results compared with urgent surgery in an unstable patient, this discrepancy may have narrowed any potential difference between the patency of arterial versus vein grafts, but this does not affect the discrepancy between current and historical reports of vein graft patency. From the optional angiograms, we ${ }^{15}$ previously reported patency of SV grafts of $82.4 \%$ at 5 years, but this included vein grafts performed to second-, third- and fourth-order coronary targets, with a reduction in the mean patency owing to inclusion of smaller caliber coronary vessels or smaller territory of runoff in lesser targets. When the SV is used only to the second best target, patency is better $(90 \%$ at 5 years on optional angiograms, ${ }^{15} 80 \%$ at 9 years in the protocol directed angiograms presented above).

The most appropriate method of analysis in this study of angiographic patency is, and has been, a source of debate. Standard conduct in a randomized trial is to analyze by intention to treat, and we have followed this convention, acknowledging that analysis by conduit used rather than assigned compromises the principles of randomization as a means of removing bias. However, in group 1 there were 9 grafts randomized to RITA that instead received

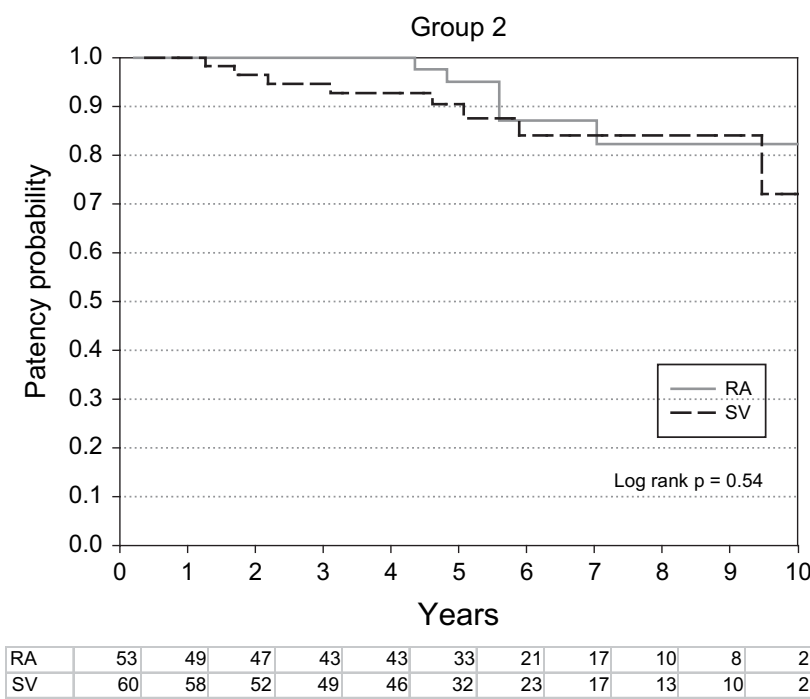

FIGURE 3. Kaplan-Meier estimates of graft patency in group 2 by intention to treat. $R A$, Radial artery; $S V$, saphenous vein.

an RA, 4 of which failed. This $44 \%$ failure rate among crossovers in group 1 favors the RA over the RITA because intention-to-treat principles allocates the 4 failures to the RITA cohort. The described absolute difference in patency in group $1(6.6 \%)$ disappears if intention to treat is not used.

\section{CONCLUSIONS}

Comparable angiographic and clinical outcomes at mean 5- to 6-year follow-up can be achieved with any free arterial graft in patients aged less than 70 years, and with an RA or $\mathrm{SV}$ in older patients, when grafted to the largest non-LAD target. This finding allows the surgeon considerable flexibility in the selection of conduits according to other patient comorbidities or factors, when 5-year outcome is envisaged. Whether the free arterial grafts are equivalent or superior to SV grafts in younger patients is not known, and caution should be expressed in extrapolation of patency results from second-order coronary targets to lesser targets, although the likely clinical impact of graft failure in the territories of lesser targets diminishes accordingly. Graft patencies of all conduits beyond 5 years are excellent and are superior to those reported in historical controls. We have previously reported excellent survival and event-free survival in participants enrolled into our randomized controlled trial: confirmation of graft patency in these patients underpins clinical outcomes and reassures patient, cardiologist, and surgeon alike.

\section{References}

1. Serruys PW, Ong AL, van Herwerden LA, Sousa JE, Jatyene A, Unger F, et al. Five-year outcomes after coronary stenting versus bypass surgery for the treatment of multivessel disease. The final analysis of the Arterial Revascularization Therapies (ARTS) randomized trial. J Am Coll Cardiol. 2005;46:575-81. 
2. SoS Investigators. Coronary artery bypass surgery versus percutaneous coronary intervention with stent implantation in patients with multivessel coronary artery disease (the Stent or Surgery trial): a randomized controlled trial. Lancet. 2002; 360:965-70.

3. Hayward PAR, Moten S, Gordon I, Matalanis GM, Hare D, Buxton BF. Does choice of arterial graft influence long-term clinical outcomes after coronary revascularisation? Results of a radial artery versus right internal thoracic artery trial. Ann Thorac Surg. 2007;84:493-7.

4. Hayward PAR, Hare DL, Gordon I, Buxton BF. Does use of an arterial or venous conduit for the second graft alter long term clinical outcome after CABG? Results of a randomized trial of radial artery versus saphenous vein. Eur J Cardiothorac Surg. 2008;34:113-7.

5. Buxton BF, Raman JS, Ruengsakulrach P, Gordon I, Rosalion A, Bellomo R, et al. Radial artery patency and clinical outcomes: five-year interim results of a randomized trial. J Thorac Cardiovasc Surg. 2003;125:1363-71.

6. Blackstone E. Generating knowledge from information, data and analysis. In: Kouchoukos NT, Blackstone EH, Doty DB, Hanley FL, Karp RB, eds. Chap 6 Kirklin/Barratt-Boyes textbook of cardiac surgery, Vol 1. Philadelphia: Churchill Livingstone; 2003. p. 310.

7. Turnbull BW. The empirical distribution function with arbitrarily grouped censored and truncated data. J R Stat Soc B. 1976;38:290-5.

8. Finkelstein DM. A proportional hazards model for interval censored failure time data. Biometrics. 1986;42:845-54.

9. Law C, Brookmeyer R. Effects of mid-point imputation on the analysis of doubly censored data. Stat Med. 1992;11:1569-78.

10. Lytle BW, Blackstone EH, Loop FD, Houghtaling PL, Arnold JH, Cosgrove DM, et al. Two internal thoracic arteries are better than one. J Thorac Cardiovasc Surg. 1999; 117:855-72.

11. Taggart DP, D'Amico R, Altman DG. Effect of arterial revascularization on survival: a systemic review of studies comparing bilateral and single internal mammary arteries. Lancet. 2001;358:870-5.

12. Goldman S, Zadina K, Moritz T, Ovitt T, Sethi G, Copeland JG, et al. Long-term patency of saphenous vein and left internal mammary artery grafts after coronary artery bypass surgery: results from a Department of Veterans Affairs Cooperative Study. J Am Coll Cardiol. 2004;44:2149-56.

13. Fitzgibbon GM, Kafka HP, Leach AJ, Keon WJ, Hooper D, Burton JR. Coronary bypass fate and patient outcome: angiographic follow up of 5,065 grafts related to survival and reoperation in 1,388 patients during 25 years. J Am Coll Cardiol. 1996;28616-26.

14. Campeau L, Enjalbert M, Leasperance J, Bourassa MG, Kwiterovich P Jr, Wacholder $\mathrm{S}$, et al. The relation of risk factors to the development of atherosclerosis in saphenous vein bypass grafts and the progression of disease in native circulation: a study 10 years after aortocoronary bypass surgery. N Engl J Med. 1984; 311:1329-32.

15. Hayward PAR, Buxton BF. Angiographic patency of coronary artery grafts: 5year observational data from the randomized controlled Radial Artery Patency and Clinical Outcomes (RAPCO) Study. Ann Thorac Surg. 2007;84:795-9.

\section{Discussion}

Dr Stephen E. Fremes (Toronto, Ontario, Canada). I do not have any personal conflicts of interest to declare.

This study reports on a 5-year patency of RA, free RITA, and SV grafts to non-LAD targets. The study is remarkable in that more than 600 patients were recruited for a surgical trial, all from a single center. The study has been performed with great rigor, with few protocol violations or crossovers, with excellent follow-up, and with a high rate of late angiography. Putting this study into context, the study comes from a center with a long history in angiographic and clinical outcomes of coronary surgery, as well as the influence of arterial conduits on patency.

Again, this study is, in reality, two separate trials, one comparing the free RITA with the RA in younger patients and the second comparing RAs with the SV in older patients. The former study is truly unique and to my knowledge is the first and only randomized controlled trial comparing a free RITA and an RA. I would like to em- phasize, however, that the authors are reporting 5-year results, and it should be emphasized again that according to protocol, the angiographic follow-up of the study is weighted toward the sixth to tenth postoperative years. I have a few questions.

First, the manuscript says that the primary analysis was performed according to a treatment received rather than an intentionto-treat basis. Why would you do that when it is more likely to introduce bias in terms of your results?

Dr Hayward. Thank you for your question, Dr Fremes. We had a lot of discussions about whether the analysis should be by intention to treat. We reported last year the clinical outcome data, which, as in any clinical trial, were analyzed by intention to treat, and we therefore initially thought that we should follow that and report the patency by intention to treat. However, it seemed a little curious to report the patency of what we know to be an SV to a given target, for example, as if it were an RA, as intended. The conduit is known, the target is known, and if the crossover was for a legitimate reason, its patency is a fact, related presumably to the conduit used, whatever was planned. The trial is intended as a pragmatic trial to guide surgeons. The surgeon wants to know, "If I place a saphenous vein to this marginal, what will the patency be?", rather than "What would the patency be if I planned to perform a radial, but didn't?', So after some discussion we decided to follow the conduit used.

However, I agree that you can argue it either way. We did in fact perform the analysis by intention to treat as well just for a statistical cross-check, and actually it does not influence the results one iota. But I agree that you could make this criticism of our decision.

Dr Fremes. I think if there are a lot of crossovers, then it is very difficult to know what to do, but you had very few crossovers or protocol violations.

Have you identified any patient or vessel predictors of graft failure overall or for the different types of grafts?

Dr Hayward. In this subset, no, we have not, because we have not asked the question. We decided that because the number of graft failures thus far has been relatively small, we would be playing with such small numbers that it would be meaningless. Thus we have not yet done the sort of analysis that you published for your cohort last year. That will come when we have the full 10-year data.

Dr Fremes. I suspect that several patients have likely undergone serial angiography in your study. Do you have any insights that may be relevant from these patients?

Dr Hayward. From an article we published last year, we did report a small number of patients that have had serial angiograms. They were patients who had the protocol-directed angiogram and then opted for the 5-year angiogram as well, and there were only two changes, or two failures, in cases that had previously been reported. Therefore, there was a $99 \%$ correlation. The groups that I have just presented today contain only the protocol-directed angiograms. We took the last angiogram as the end point, effectively. The moment a graft failed, it remains failed whatever a future angiogram may suggest, or if for some reason they had two angiograms and both have shown the graft as patent, we have taken the longer follow-up, for obvious reasons.

Dr Fremes. That is fine for the results, but were there any sequential findings that may give you some insights as to what might happen if there is longer-term follow-up?

Dr Hayward. Not yet at this point, no. Not enough patients have had two angiograms. 
Dr Fremes. One of the surprising results was the patency of the SV. The patients were recruited largely from the 1990s as opposed to some more recent studies, for example, the PREVENT IV, which have shown much poorer results. Can you speculate as to the possible reasons for this disparity?

Dr Hayward. I think ultimately the two factors have been surgical technique and secondary prevention. I think secondary prevention has been absolutely rigorous in that all patients have been monitored by their hospital cardiologist, and the standard of practice is extremely high in Australia. I agree that some of the patients were operated on at a time before with what we might regard as the latest preventative strategies, but all the patients received appropriate medications from surgery onward.

More important, though, is the surgical technique. The rigor with which high-quality conduit is selected in Melbourne is very significant, in my opinion, and the delicacy and respect with which it is handled is also striking. Because there is such concern about the quality, often large amounts of conduit are harvested and only a smaller segment used. I think it is that selectivity that has made the difference here.

Dr Fremes. I have one final question, and you have alluded to this a bit already: were there clinical events attributable to study graft failure and how did they compare between the different grafts?

Dr Hayward. We do know the clinical events and published these last year, and we do know the patients with the study graft failures. In the 10-year results we are going to correlate those two. But in the current patency data we did not attempt to link the patients from the two presentations, because at the moment there have only been clinical events in about $10 \%$ of patients, and there are only a small number of failed grafts. Thus there really are not enough to draw any conclusions at this time. I have deliberately not done that. The data are there, but I think we should wait until we have more graft failures.

Dr Thomas Z. Lajos (Greenwood, SC). I have no conflicts of interest. I would like to congratulate the authors for this very excellent study. I would like to make a comment and a question.

We investigated veins, reverse veins, with valves and without valves, 10 years ago, and we found that if you take the best quality veins on both sides, the longer patency is present in those that have no valves. So it is a valveless vein. Their patency is about the same as you have shown, and we found the same thing; with the veins, when the follow-up starts to decrease, patency is in the end of 9 to 10 years. The valves in the veins create a dilatation, they create turbulence, and they may create emboli and thrombus inside. Did you discard those veins or you did you have a chance to discard those veins that had valves versus no valves?

Dr Hayward. Veins were not necessarily discarded per se because they had valves, but because of the practice of selecting the very best conduit. Where possible, lengths are trimmed between the valves. So as far as possible we would have valveless segments of vein. That, of course, is not achievable in all patients. But I agree that no doubt the turbulence at those points is a major factor. Sometimes dilatations or outpouchings can be flattened off with a large clip to try to make a smoother profile, but that does not remove the disturbance of flow imposed by a valve. I would say that valves are avoided where possible, but I would not say that this is a series of valveless veins. That would be misleading. However, I certainly agree with your conclusions.
Dr Georg Lutter (Kiel, Germany). Congratulations for your good patency rate of your RA. Can you give us a little insight of whether you applied some vasodilating substances to your RAs? A second question might be whether you dissected them. Did you take the fascia, the small thin layer of the fascia off, or did you leave the conduit in place as you took it out from the lower arm?

Dr Hayward. The RAs in our unit and by other surgeons in Melbourne are harvested by a very standardized technique that the senior surgeons locally developed. One of the important design features of the trial was that they standardized everything that they could with the conduits. The RA is taken as a pedicle, a relatively narrow pedicle, but the artery is never touched at all, and the only manipulation is of the fascia. The artery is divided early on distally and then retrogradely injected with a solution of papaverine dilated in blood and Ringer lactate 50/50, and that same solution is used for the veins as well. The graft is then clipped off at the distal end and the dissection of the pedicle is then completed while the graft is allowed to dilate with normal blood pressure; only at the last minute is it removed from the proximal end. Then it is placed in a bath of papaverine and blood and Ringer lactate. All the conduit types were treated in the same manner.

Dr Thoralf Sundt (Rochester, Minn). I suspect I know the answer to this question, but there has been speculation that the poor vein graft patency in the PREVENT IV trial was due to endoscopic harvest. Were any of these veins harvested endoscopically, and could you make a comment about the potential impact of that innovation on graft patency?

Dr Hayward. All the veins were harvested by an open technique here. I think that the delicacy with which the conduit is handled in Melbourne would make an endoscopic approach to this very difficult. Many of us who have had any experience, and in my case relatively limited experience, with endoscopic harvest have been uncomfortable with the amount of traction I or we have placed on the vessels. Endothelial integrity is absolutely key to these patency results, and this integrity might have been threatened by an endoscopic method. These veins were all taken in a shamelessly open no-touch manner.

Dr Charles C. Canver (Riyadh, Saudi Arabia). Congratulations on your outstanding work. I think you, Dr Buxton, and your other group members have contributed immensely to our understanding of the clinical use of the RA as a conduit. Most of the experienced surgeons sensed the need for the RA during coronary artery bypass grafting, but most were very skeptical owing to its vasospastic nature. Accordingly, it is gratifying to see that your results support that the RA is probably the second best arterial conduit. I have a couple of questions for you.

First, have you ever used the RA as a sequential graft or have you simply used the RA to a single coronary target? Second, if the patients had insulin-dependent diabetes, did you validate the quality of the in situ RA? Last, have you used any preservative solutions to prevent RA spasm just before its use in the operating room, that is, a mixture of papaverine, nitroglycerin, diltiazem, or whatever? In addition, did you use any perioperative intravenous vasodilators, that is, diltiazem, nitroglycerin, or milrinone?

Dr Hayward. Thank you. I will take your questions in reverse order. On the table, as I said, after harvesting, the conduit is in a papaverine-blood-Ringer lactate bath and is kept flushed with this 
solution. In terms of intravenous agents, at completion of the procedure the patients at Austin are given low-dose milnirone as an arterial graft dilator, and that is run for about 24 hours unless there is a reason to discontinue it, such as low systemic vascular resistance state or excessive cardiac output. The use and dose of the milnirone is entirely vasodilating in its intent rather than inotropic. I think that is an important factor in the treatment of arterial grafts in this series.

A substantial number of the patients did have diabetes. Type II diabetes was predominant, although type I diabetes was present in about $15 \%$ of those with diabetes. The patients were all assessed by a preoperative Allen test, and then intraoperatively, before use, the RA was inspected and palpated for calcification. After harvesting, if the internal lumen showed evidence of atheroma or calcification, then it was discarded. There was no Doppler study, if that is what you were referring to, but a fairly rigorous visual inspection and palpation were made of the conduit.

Your final question had to do with sequential grafts. All of these are grafts with single distal anastomoses. There were a few patients who received sequential grafts, but they were then eliminated from the study, as shown on the flow diagram. We are not opposed to sequential grafting, and in fact, a proportion of the LITAs were sequentially grafted. I think there is a growing body of opinion that if you double the territory of runoff for the graft, you may in fact improve its patency, but that could bias your patency data. For the purposes of this trial, to keep it simple and reproducible, the randomized or study conduit had to be used with one distal end, and if used sequentially with a larger bed of runoff, it was eliminated from the study. 